\title{
ASYMMETRIC B-FACTORY COLLIDER NOTE
}

\section{TITLE: SYNCHROTRON RADIATION LEAKAGE FROM THE B-FACTORY BEAM PIPE}

\author{
AUTHORS: T. M. Jenkins, W. R. Nelson and N. Ipe.
}

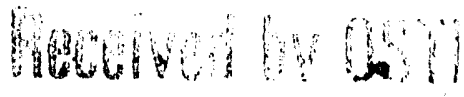

DATE: September 20, 1990

NOV 281990

\section{General.}

The high-energy ring (HER) of the B-Factory, running at an energy of $9 \mathrm{GeV}$, generates the synchrotron spectrum shown in Fig. 1 when applied to a ring with the PEP bending radius $(165 \mathrm{~m})$. The $\mathrm{B}$-Factory HER may also run at $12 \mathrm{GeV}$ (though this is not the original design goal), producing the harder spectrum shown in Fig. 2. Depending upon beam-pipe material and thickness, some of this radiation may escape and deposit energy in the surrounding material. This was originally pointed out in PEP-109 ${ }^{(1)}$ during the initial design of PEP, and subsequently verified by measurements at both PEP and PETRA at DESY. Of concern to the $\mathrm{B}$ Factory is magnet insulation, though other adjacent materials such as wire insulation and cooling water hoses are even more radiosensitive.

Radiation damage to magnets is a function of the type of material used in the potting compound. The PEP magnets, which hopefully can be used for the bigh-energy ring of the B-Factory, are insulated with an epoxy composed of DER-332, DER-732, NMA and aluminum oxide. It is estimated that this epoxy compound should tolerate doses near the $10^{10}$ rad range. The exact number isn't known, so we rely upon other sources to set a criterion. CERN used $3 \times 10^{9}$ rads as the dose criterion for the LEP magnets. ${ }^{(2)}$ They note that using fiber glass tape in the insulation gives a factor of ten higher tolerance (i.e., in the $10^{10}$ region). We have elected to use $3 \times 10^{9}$ rads as a conservative criterion for the B-Factory magnet insulation. Put another way, the criterion will be $10^{8}$ rads per year; this should give a magnet lifetime of 30 years or more (in this note, we ignore doses already received by the PEP magnets from PEP running to date.)

To summarize the results of these calculations, $0.87 \mathrm{~cm}$ of copper is needed at the point of maximum dose from $12-\mathrm{GeV}$ operation in order to reach the dose criterion if a rectangular beam pipe is used. The copper needs to be only $0.7-\mathrm{cm}$ thick for an octagonal beam pipe and the same energy. For $9-\mathrm{GeV}$ operation, an octagonal copper pipe needs only to be $0.25 \mathrm{~cm}$ thick. An octagonal aluminum pipe that is $0.5-\mathrm{cm}$ thick also needs $0.3 \mathrm{~cm}$ of lead on the outside to reach the same criterion for $12-\mathrm{GeV}$ operation. For $9-\mathrm{GeV}$ operation, the aluminum pipe still requires a lead liner. These thicknesses are at the point of maximum dose only (top and bottom of the beam pipe)-i.e., the rest of the pipe can be thinner.

\section{MASTER}

OSTRIBUTION OF THIS DOCUMENT IS UMLIMUS 


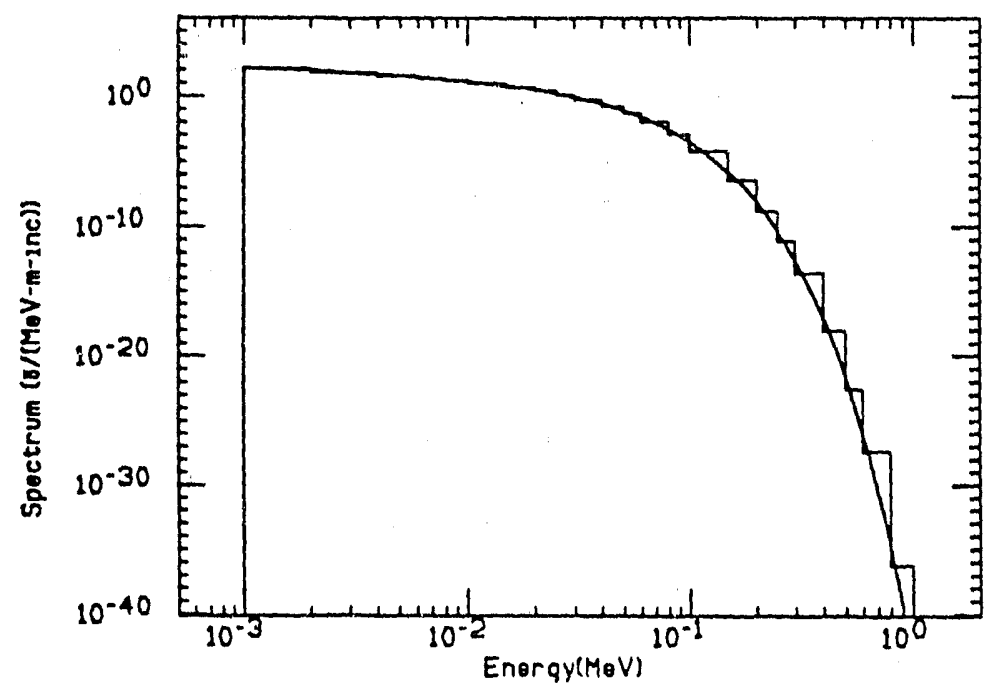

Figure 1. Synchrotron spectrum from 9-GeV operation at PEP. Solid line $=$ analytic; histogram $=$ EGS sampled spectrum.

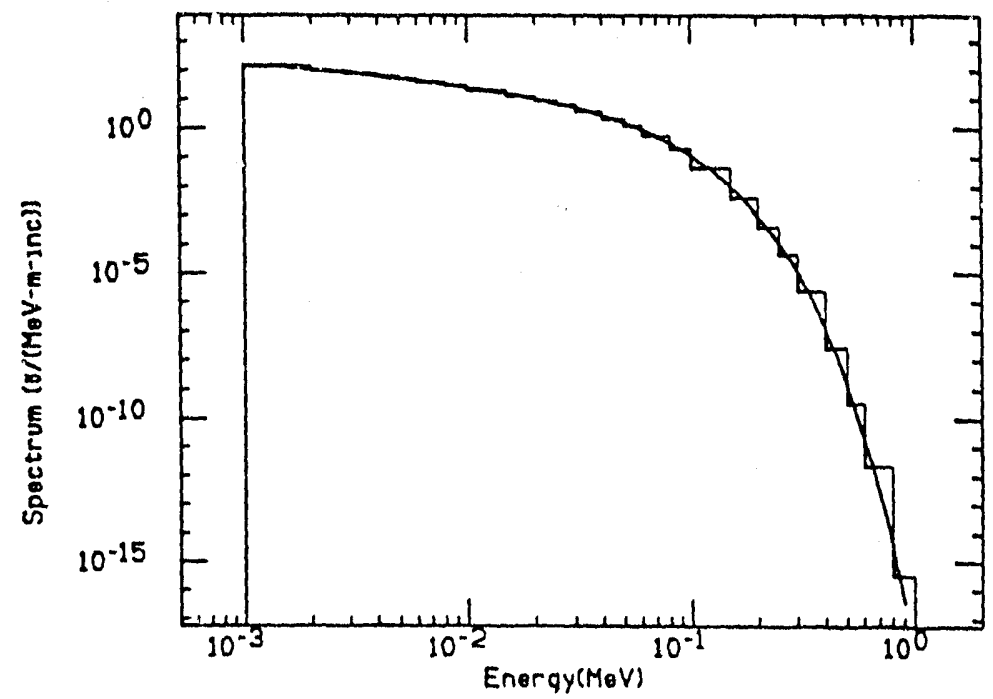

Figure 2. Synchrotron spectrum from $12-\mathrm{GeV}$ operation at PEP. Solid line = analytic; histogram = EGS sampled spectrum.

\section{DISCLAIMER}

This report was prepared as an account of work sponsored by an agency of the United States Government. Neither the United States Government nor any agency thereof, nor any of their employees, makes any warranty, express or implied, or assumes any legal liability or responsibility for the accuracy, completeness, or usefulness of any information, apparatus, product, or process disclosed, or represents that its use would not infringe privately owned rights. Reference herein to any specific commercial product, process, or service by trade name, trademark, manufacturer, or otherwise does not necessarily constitute or imply its endorsement, recommendation, or favoring by the United States Government or any agency thereof. The views and opinions of authors expressed herein do not necessarily state or reflect those of the United States Government or any agency thereof. 


\section{Machine Parameters and the EGS4 User Code}

For circulating beam current, we use $1.5 \mathrm{~A}$ for a 6000 hour operating year ${ }^{[3]}$, giving $9 \times 10^{3}$ $A-h / y$. The absorbed dose, $D$, in rads/electron, is

$$
\begin{aligned}
\mathrm{D} & =\frac{10^{8} \frac{\mathrm{rad}}{y} \cdot \frac{1.6 \times 10^{-19} \mathrm{~A}-\mathrm{sec}}{\mathrm{e}^{-}}}{9 \times 10^{3} \frac{\mathrm{A-h}}{\mathrm{y}} \cdot 3600 \frac{\mathrm{sec}}{\mathrm{h}}} \\
& =4.94 \times 10^{-19} \mathrm{rads} / \mathrm{e}^{-}
\end{aligned}
$$

This number is used when applying EGS4 to the problem, which will give answers per incident electron, either in terms of fluences, energy deposited, or rads (using appropriate conversion factors). Various user codes, beginning with the simple UCSR006 and progressing through UCSR28 and UCSR28CG (CG = Combinatorial Geometry), were developed specifically to study this problem. These codes all generate the synchrotron spectrum both analytically and from EGS sampling (see Figs 1 and 2) with scoring done in the regions outside the beam pipe. A tluence-to-dose conversion, using the surface dose numbers of Rogers, ${ }^{[4]}$ modified for the SLAC epoxy compound, is performed within the code each time the epoxy region is entered.

Calculations are based on the configuration of the present PEP ring which has a magnet bending radius of $165 \mathrm{~m}$ inside a $2200 \mathrm{~m}$ circumference tunnel. Only the high-energy ring (HER) is considered; synchrotron radiation won't penetrate the beam pipe of the low-energy ring (LER) which runs at $3 \mathrm{GeV}$.

The following is a brief summary of pertinent B-Factory HER parameters:

- 1.5 A circulating current.

- $165 \mathrm{~m}$ bend radius (synchrotron radiation strikes at $1.7^{\circ}$ ).

- $12 \mathrm{GeV}$ maximum energy (9 GeV nominal).

- $10^{8} \mathrm{rads} / \mathrm{y}$; for calculational purposes, this is about $5 \times 10^{-19} \mathrm{rad} / \mathrm{e}^{-}$.

- 6000 hours per year operation.

The chemical makeup and weight in grams of the epoxy used in the PEP magnets is:

- DER 332 - epichlorohydrin + bisphenol $\mathrm{A}\left(\mathrm{C}_{21} \mathrm{H}_{24} \mathrm{O}_{4}\right)=851 \mathrm{~g}$

- DER 732 - epichlorohydrin-polyglycol $\left(\left(\mathrm{C}_{3} \mathrm{H}_{6} \mathrm{O}\right)_{\mathrm{n}} \mathrm{C}_{6} \mathrm{H}_{10} \mathrm{O}_{3}\right)=1049 \mathrm{~g}$

- NMA - nadic methyl anhydride $\left(\mathrm{C}_{9} \mathrm{H}_{8} \mathrm{O}_{3}\right)=1832 \mathrm{~g}$

- Aluminum oxide $\left(\mathrm{Al}_{2} \mathrm{O}_{3}\right)=4252 \mathrm{~g}$

* All EGS4 user codes are written in Mortran3 and, together with the necessary input data, are maintained (archived) in the TMJRP account under SLAC VM. 
The weight fractions of the elements making up the epoxy (for EGS4 purposes) are:

- Hydrogen $=2.6 \%$

- Oxygen $=38.4 \%$

- Carbon $=30.7 \%$

- Aluminum $=28.3 \%$

A new PEGS4 data set (BFLOW DATA; was generated for photons down to $1 \mathrm{keV}$ and electrons to $10 \mathrm{keV}$ ( $521 \mathrm{keV}$ total energy), though for most calculations the actual cutoffs were raised to $10 \mathrm{keV}$ (photons) and $1 \mathrm{MeV}$ (electrons). Upper energies for both electrons and photons were $10 \mathrm{MeV}$, which is adequate since the the spectrum is essentially zero above a few $\mathrm{MeV}$ for both 9 and $12 \mathrm{-GeV}$ electron energies. The photon spectrum was sampled uniformly within an energy range (i.e., 10 times above and below the critical energy), and a weight carried along with each photon (and progeny) for scoring purposes. The final results were later normalized per incident beam electron.

The initial runs to check the code were done in simple Cartesian geometry (UCSR006) using only top and bottom slabs (no end pieces or water cooling compartment) in order to compare with previous PEP calculations (i.e., PEP-109). To our surprise, the values from UCSR006 were found to be a factor of 6 higher than the PEP-109 numbers! The differences were tracked down in part to a PEP-109 error in the method of scoring fluences, where all photons were assumed to cross the scoring plane at $90^{\circ}$-i.e., not dividing by the cosine of the angle of incidence relative to the normal to the surface results in numbers that are low by about a factor of about 2. The other factor of 3 comes from having used tissue as the scoring medium in the PEP-109 note versus epoxy in the new calculations. Both are handled correctly in the new user codes.

\section{Rectangular beam pipe geometry}

Initial calculatious using the new code were made with a rectangular beam pipe having a single water channel in the direction of the synchrotron radiation (outer edge of the beam pipe). An outer liner was included in the geometry, which is set to $\mathrm{Pb}$ when the beam pipe material is aluminum, and vacuum when the beam pipe material is copper. The EGS4 representation of this geometry is shown in Fig. 3. Though this isn't the actual PEP configuration, which is somewhat oval or octagonal in cross-section, the results of the calculations are informative and will be included here. In this geometry, which has the $\mathrm{X}$-direction to the right and $Y$ up, the synchrotron beam is incident on the beam pipe at the right side just before the water channel. The beam pipe thichness before entering the water is $0.8 \mathrm{~cm}$, the water channel is $0.8-\mathrm{cm}$ thick, and the following thickness is $0.5 \mathrm{~cm}$. The incident angle of the synchrotron radiation relative to the $Z$-direction (out of the page) is $1.7^{\circ}$. The magnet epoxy (scorint plane) is about $0.3 \mathrm{~cm}$ above the outer liner.

Using this geometry, the top and bottom beam pipe thicknesses can be varied to see the effects on the scoring plane. Typical curves of dose along the magnet epoxy plane are shown in Figs. 4 through 7 for a rectangular copper pipe using the geometry given in Fig. 3. Copper thicknesses are $0.25 \mathrm{~cm}$ (Fig. 4), $0.5 \mathrm{~cm}$ (Fig. 5), $0.75 \mathrm{~cm}$ (Fig. 6), and $1.0 \mathrm{~cm}$ (Fig. 7). The regions of interest are 1) the forward direction (through the thick copper and water channel, 2) the region directly above the vacuum pipe stay-clear and 3) the region to the rear. 


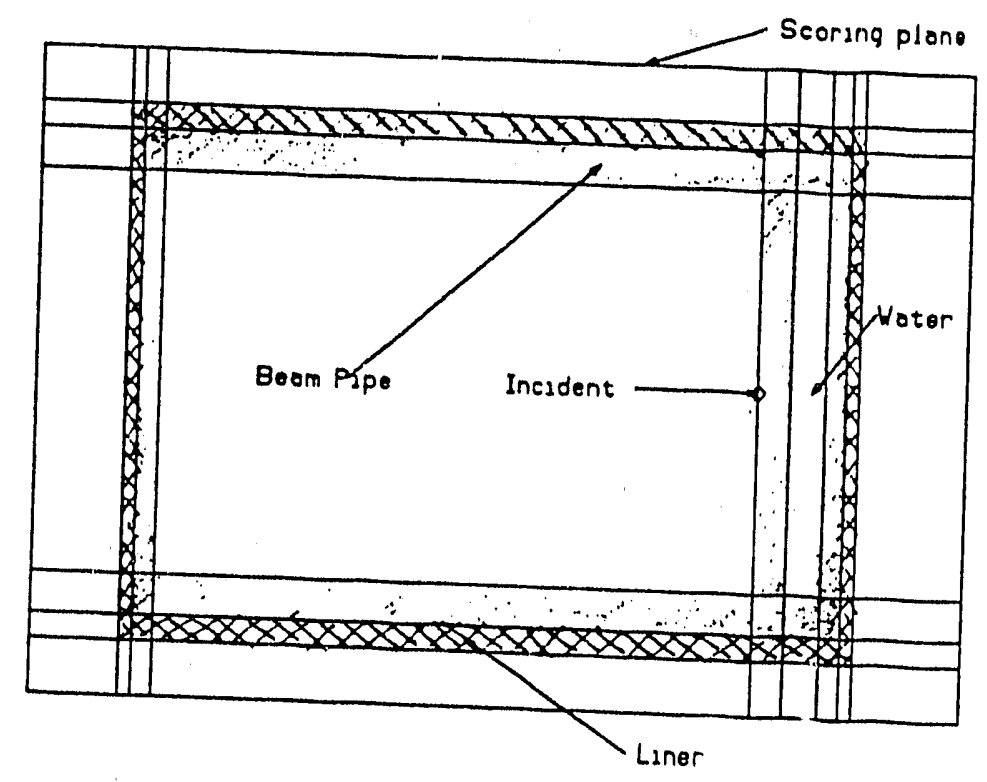

Figure 3. Rectangular beam pipe configuration for the present PEP geometry.

The low energies in the synchrotron spectrum are such that the only radiation which can escape after striking the beam pipe is that which has undergone a large-angle scatter, and into the beam pipe vacuum to be in order to get out. Thus we would expect backscattering ( $1.7^{\circ}$ in these calculations) probably isn any small angle of incidence.

The synchrotron beam-entrance location is at $\mathrm{X}=5 \mathrm{~cm}$ in these and subsequent figures. As can be seen from the curves, the dose in the forward direction (toward the outside of the ring) drops dramatically due to the relatively large amounts of copper in the water-cooled section, and more slowly in the backward direction. The maximum dose occurs slightly radiation also traverses a expected since it is the closest point from beam entry and the both the distance and the material thickness. As the backward angle increases past $90^{\circ}$, epoxy plane. Plotting the maximum dose (i.e., the dose leading to the fall-off of dose to the of copper thickness for this rectangulare (i.e., the dose around $X=3$ to $5 \mathrm{~cm}$ ) as a function 

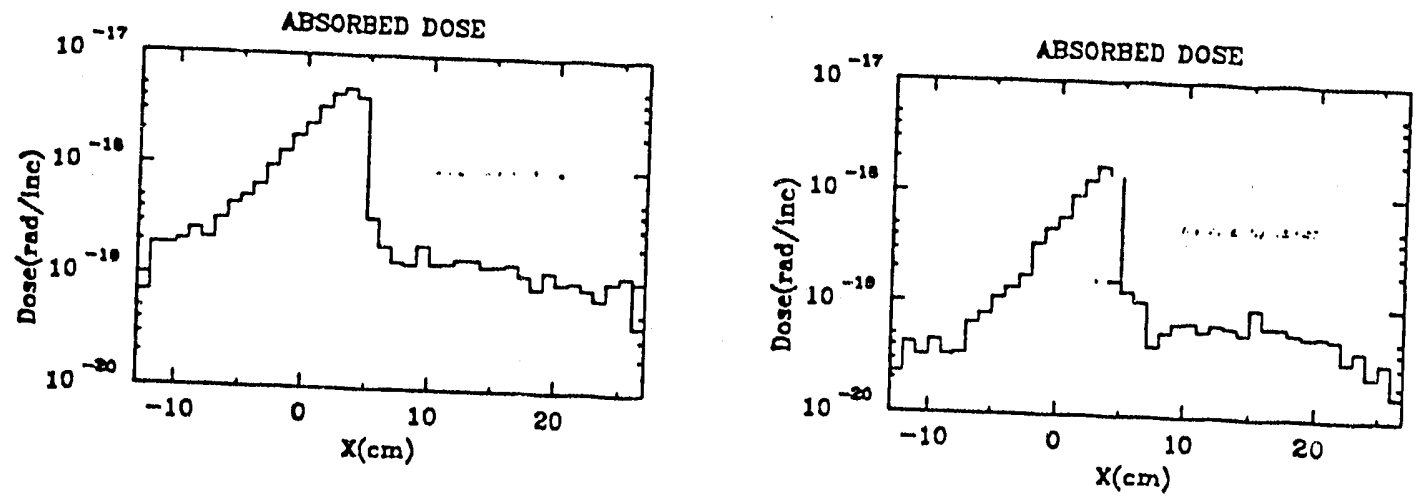

Figure 4. $0.25-\mathrm{cm}$ thick copper.

Figure 5. $0.5-\mathrm{cm}$ thick copper
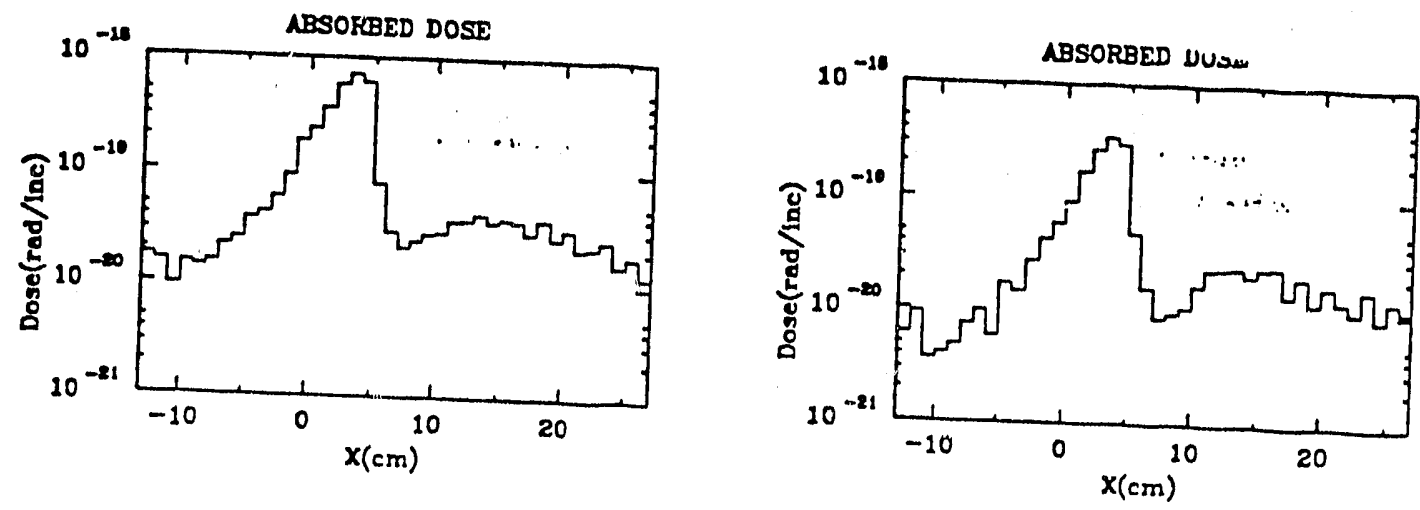

Figure 6. $0.75-\mathrm{cm}$ thick copper

Figure $7.1 .0-\mathrm{cm}$ thick copper 


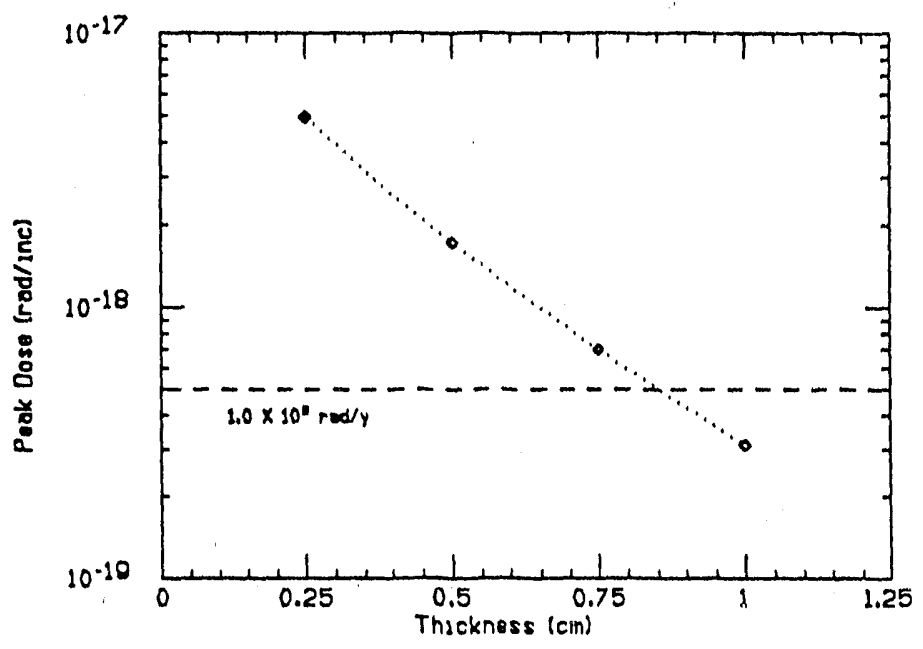

Figure 8. Maximum dose to the magnet plane epoxy as a function of copper thickness for a rectangular beam pipe.

\section{Octagonal beam pipe geometry}

The beam pipe configuration proposed for the B-Factory will be similar to the existing PEP beam pipe except for some small differences. First, the vacuum-pumping channel will be separate from the beam channel for most of the ring (the bend regions) with horizontal slots joining the two areas. Figure 9 gives a cross section view of the bend region, along with typical dimensions. The rectangular region to the left is the vacuum pumping channel, which will be missing for part of the ring (in the quad regions) as shown in Fig. 10. We have chosen to represent these areas in cross section using two different EGSA geometry options: 1) the regular (default) EGS4 geometry using cylinders and slabs where the tracking is done by the user in subroutine HOWFAR, and 2) the combinatorial geometry package, interfaced to HOWFAR, where the user defines the geometry using combinations of cylinders, boxes, etc., and lets the geometry routines do all the tracking. The normal EGS4 geometry is about $2-1 / 2$ times faster for these problems due, most probably, to the fact that the tracking is done in-line rather than making external calls to subroutines as is done with the combinatorial geometry packages. The latter is, however, much simpler to implement for complicated geometries, and is the preferred method. The EGS4 representation of the bend geometry is shown in Fig. 11 using the combinatorial geometry option. This figure also shows an outer liner which may be either $\mathrm{Pb}$ (in the case of an Al chamber) or extra copper thickness (in the case of a copper chamber it also could be vacuum). Fig. 12 is the EGS4 representation of the quad region using the normal EGS geometry and tracking routines. Synchrotron radiation is incident at $X=5 \mathrm{~cm}$ at an angle of $1.7^{\circ}$ out of the page (the Z-direction). 


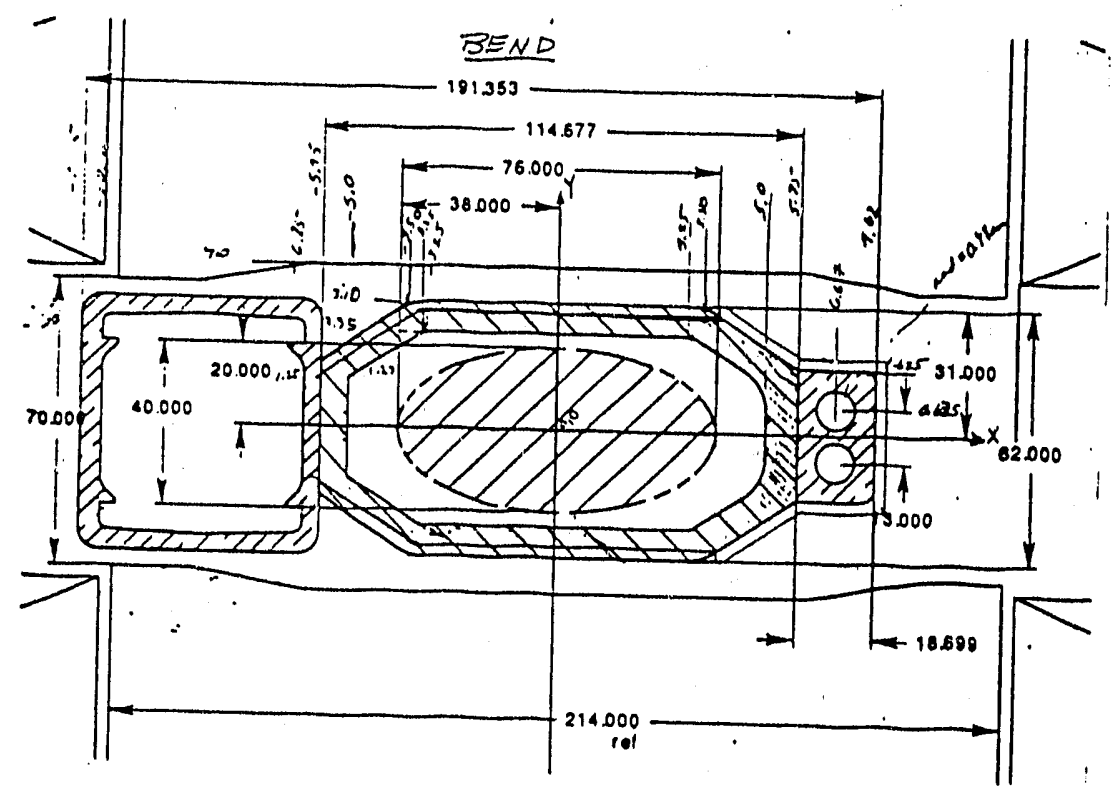

Figure 9. Proposed beam pipe configuration for the bend region.

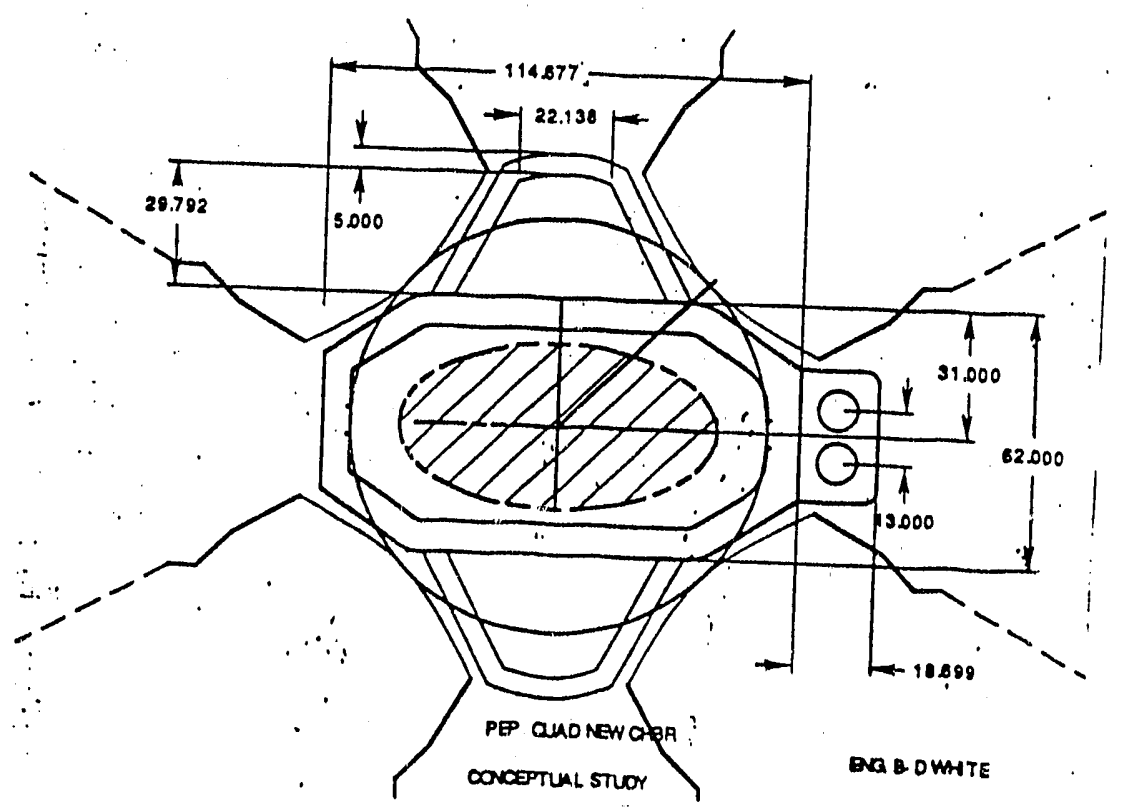

Figure 10. Proposed beam pipe configuration for the quad region. 


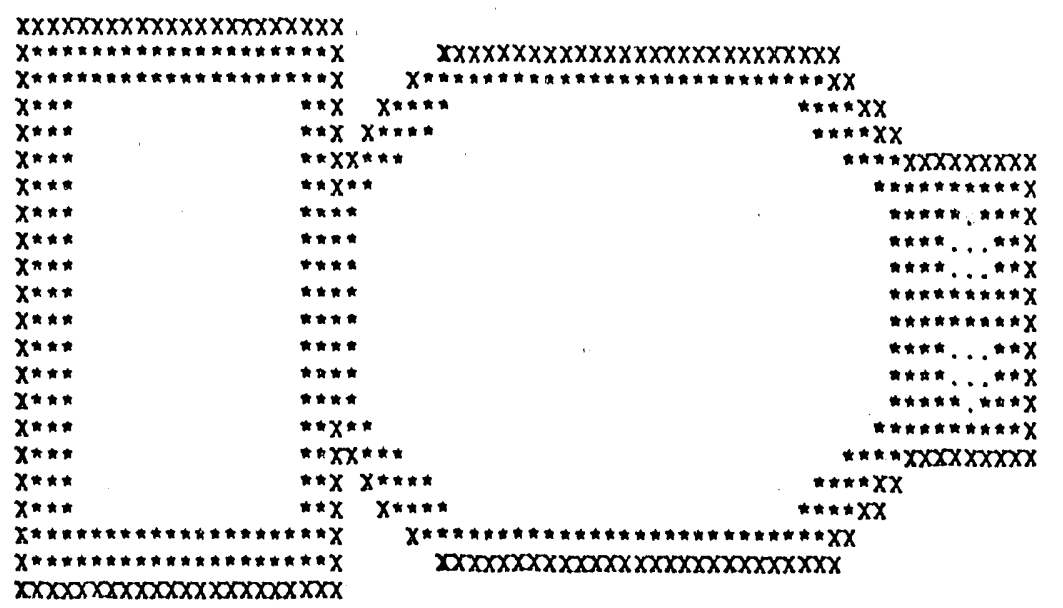

Figure 11. EGS4 representation of bend region using combinatorial geometry. Outer liner region included.

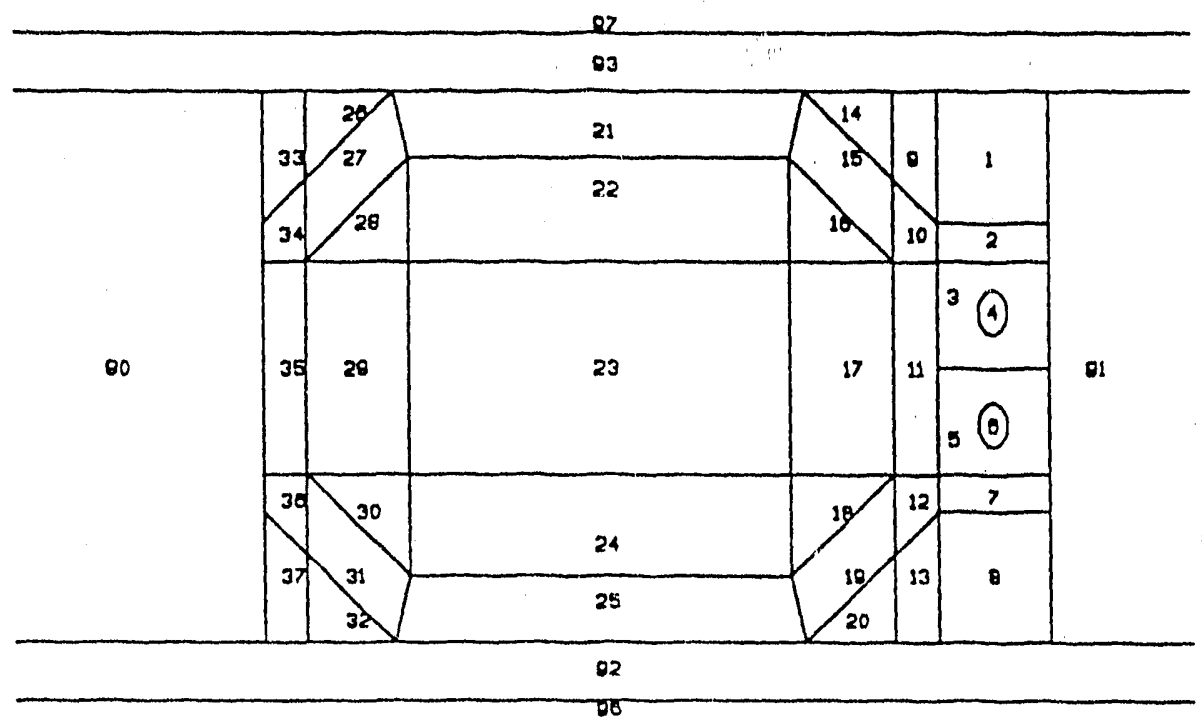

Figure 12. EGS4 representation of quad region using normal geometry. No outer $\mathrm{Pb}$ liner. 
Simply shifting to an octagonal geometry (a quad beam pipe is shown in Fig. 13 to give a better perspective, and to show some important dimensions) causes the peak transmitted doses (at $X=3$ to $5 \mathrm{~cm}$ ) to decrease due to the increased thickness the rays 'see' in the vertical directions (since they traverse the material at an oblique angle). At the same time, rays in the backward direction traverese less material when they reach the part of the pipe that is slanted. This is seen in Fig. 14 for a 0.5 -cm thick copper beam pipe.

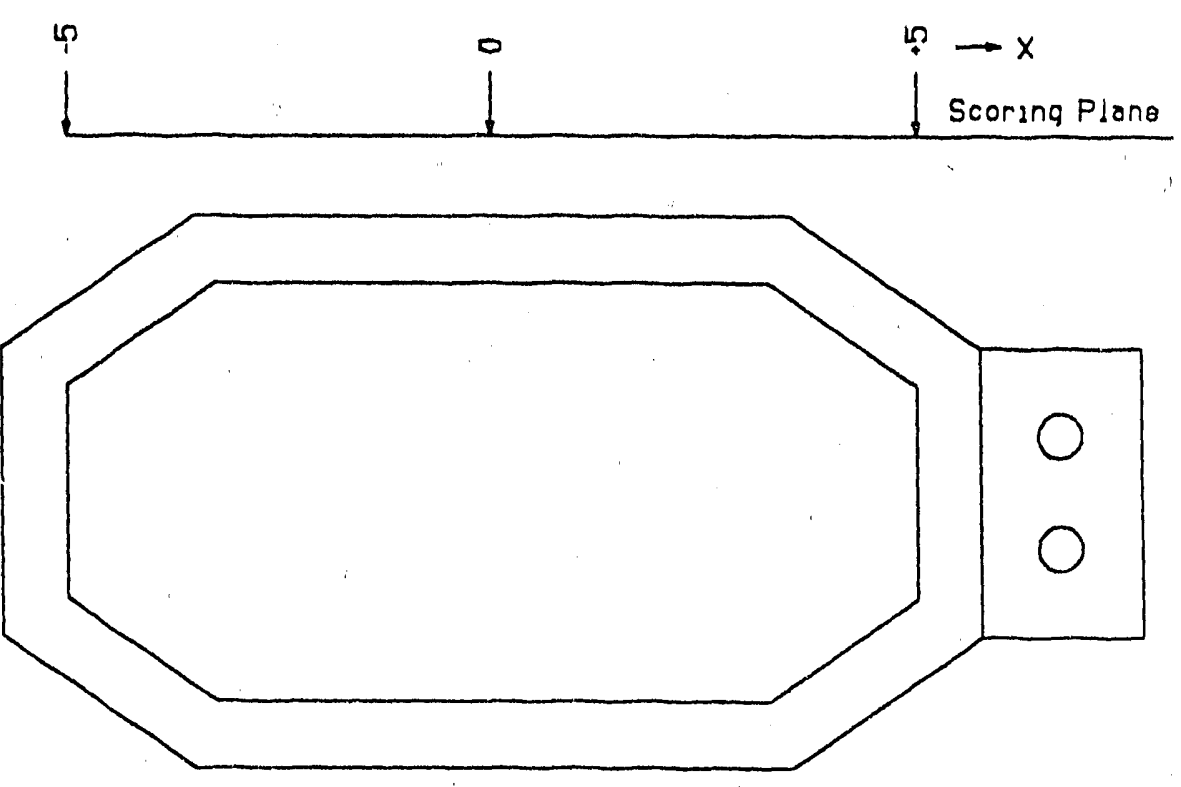

Figure 13. The octagonal beam pipe of the quad region where there is no varuum-pumping channel. The scoring region is shown above.

The peak directly above the $5-\mathrm{cm}$ beam entrance location of the previous rectangular geometry (see Fig. 5) has now been suppressed in Fig. 14 for the octagonal geometry, with a new peak located about $4 \mathrm{~cm}$ in the backward direction (at $\mathrm{X}=1 \mathrm{~cm}$ ). The dose magnitude at this point $(X=1 \mathrm{~cm})$ is about equal to that at the same location in the rectangular geometry. So the forward slant affects the region above the beam entrance, but has little effect on the horizontal portion of the beam pipe beyond the point where the slant begins.

A second peak is seen to begin at $X \leq-7 \mathrm{~cm}$ where radiation escapes through the backward slanted portion of the beam pipe in the quad beam pipe. This second peak is, however, below the criterion $\left(5 \times 10^{-19} \mathrm{rad} / \mathrm{inc}\right)$ when the beam pipe is $0.5-\mathrm{cm}$ thick in this region.

With the vacuum-pumping cavity adding shielding to backscattered radiation in the bend regions, the second region of higher doses in the backward direction is reduced significantly, as snown in Fig. 15. 


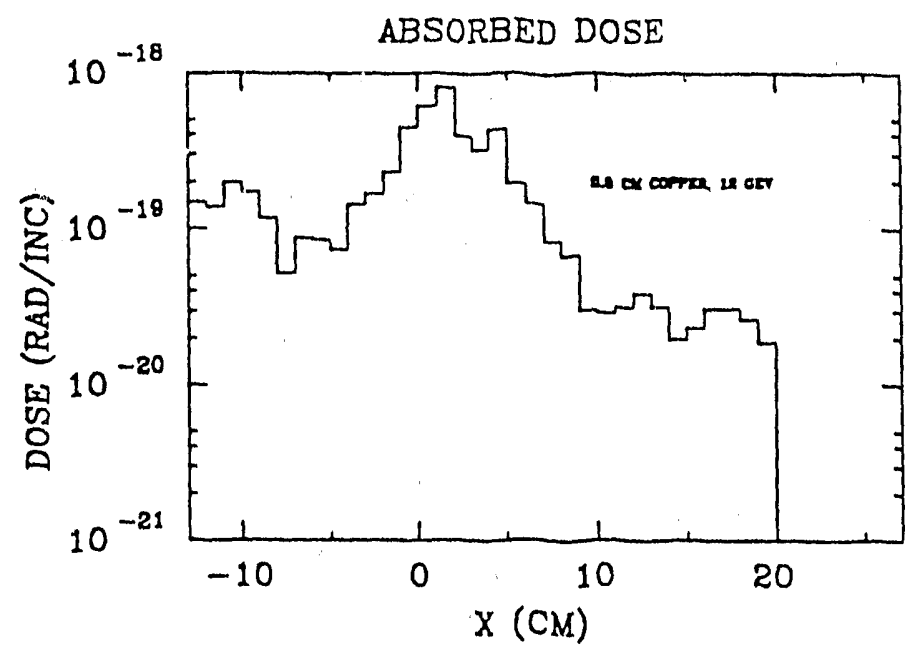

Figure 14. Dose to the magnet windings through a $0.5-\mathrm{cm}$ thick copper beam pipe with an octagonal cross section and no vacuum-pumping channel.

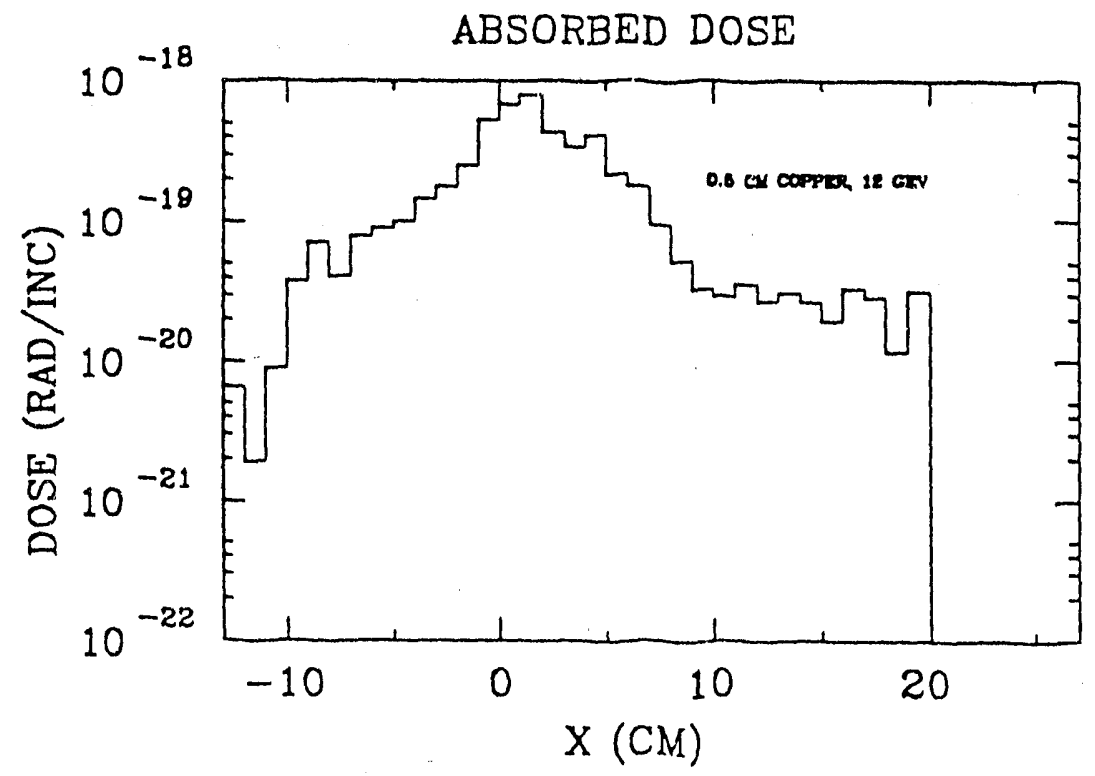

Figure 15. Dose to the magnet windings through a $0.5-\mathrm{cm}$ thick copper beam pipe with an octagonal cross section and a vacuum channel. 
A curve of the maxinum dose versus copper thickness, is shown in Fig. 16 (similar to Fig. 8), with the rectangular geometry data points included for comparison. From this figure, one can see the beneficial effects of the octagonal geometry over the rectangular geometry for the same copper thicknesses. The differences all occur in the $X=3$ to 5 -cm positions.

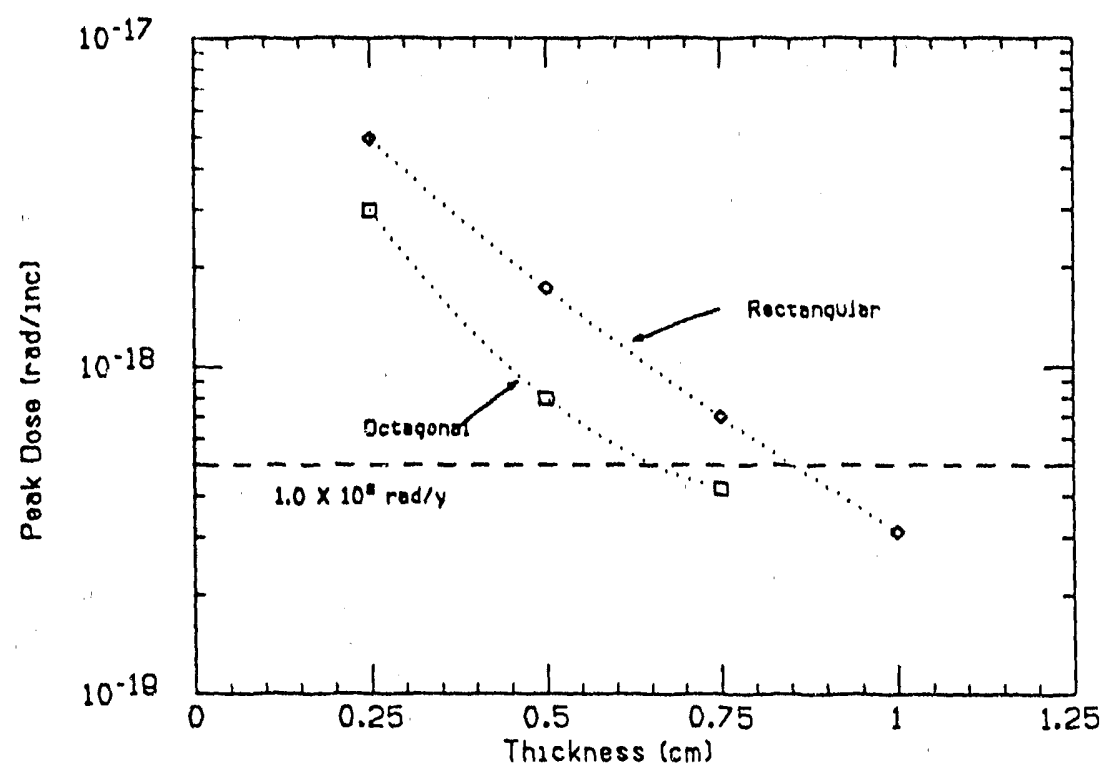

Figure 16. Dose to the magnet windings through a $0.5-\mathrm{cm}$ thick copper beam pipe with an octagonal cross section and a vacuum channel.

From these and similar curves, we show in Fig. 17 the outline of an ideal copper beam pipe whose thickness is such that it keeps doses in the scoring plane below $5 \times 10^{-19} \mathrm{rad} /$ inc $\left(1 \times 10^{8} \mathrm{rads} / \mathrm{y}\right)$. The beam pipe thickness tapers from 0.25 to $0.75 \mathrm{~cm}$ where extra copper is added. The beam pipe in the barkward direction where the slant occurs, and the vertical plane at $X=-5 \mathrm{~cm}$, should be $0.5-\mathrm{cm}$ thick. The scoring plane is shown along with the rays (dotted lines) which define the extra thickness. The rectangle containing the water channels needs only be thick enough to contain the water holes, assuming that the total copper thickness along $\mathrm{X}$ is at least $0.8 \mathrm{~cm}$. 


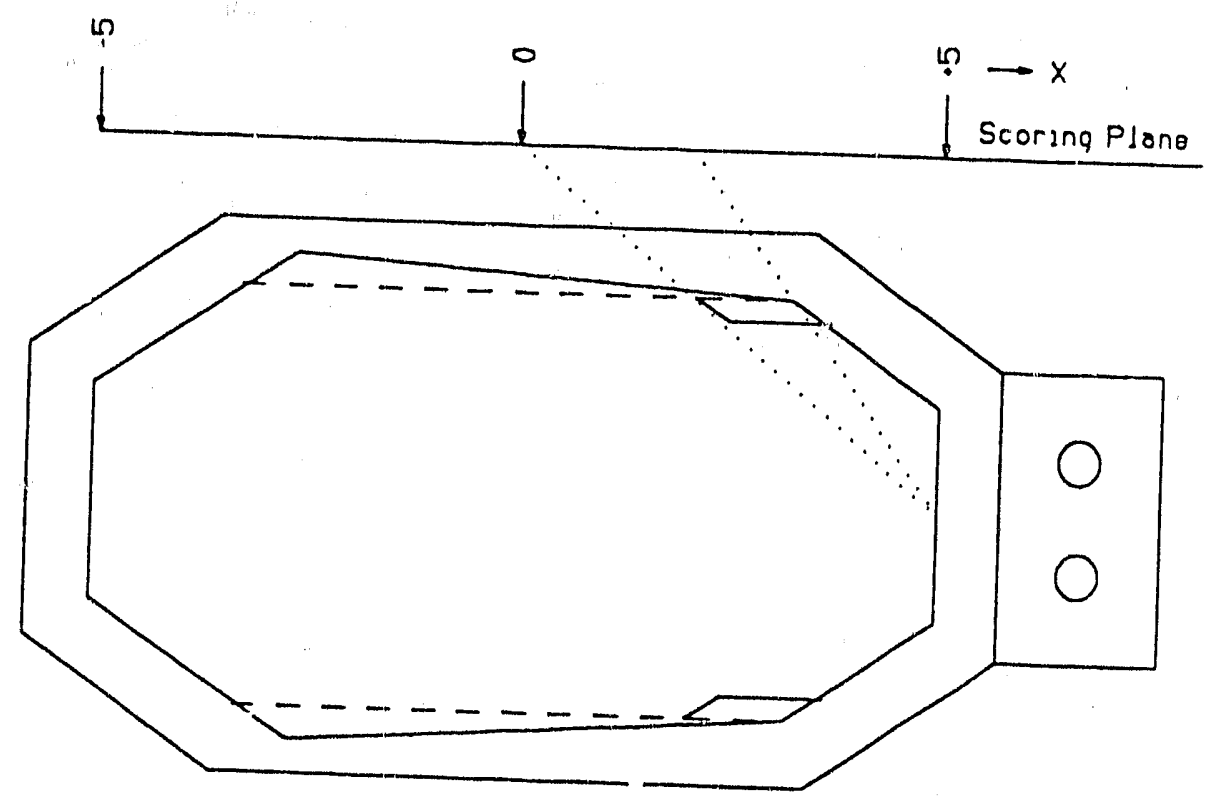

Figure 17. An idealized copper beam pipe for the B Factory in the quad region. The backward thicknesses could be thinner for the bend region due to the extra shielding of the pumping channel.

Of course we aren't suggesting that such an ideal beam pipe be built, but simply that if radiation dose to the epoxy above and nelow the beam pipe were the sole criterion, then
the beam pipe could look like this.

\section{Aluminum beam pipes and operation at $9 \mathrm{GeV}$}

Aluminum as a beam pipe doesn't absorb much of the synchrotron radiation, either at 12 $\mathrm{GeV}$ or at $9 \mathrm{GeV}$. This can be seen in Fig. 18 for $12-\mathrm{GeV}$ operation where the Al beam pipe is surrounded by a $0.25-\mathrm{cm} \mathrm{Pb}$ liner. The dose between $\mathrm{X}=0$ and $2 \mathrm{crn}$ is above the criterion; the $\mathrm{Pb}$ liner would need to be thickened in that region (i.e., $0.3 \mathrm{~cm}$ or so total). Doses without the $\mathrm{Pb}$ are many orders-of-m ignitude too high to be considered and aren't shown here for that reason. At $9 \mathrm{GeV}$ the dose to the ef Jxy through a $0.5-\mathrm{cm}$ thick aluminurn pipe without a lead liner is everywhere above the criterion $\left(5 \times 10^{-19} \mathrm{rad} / \mathrm{inc}\right)$, as seen in Fig. 19. $\mathrm{A} \mathrm{Pb}$ liner $0.25 \mathrm{~cm}$ thick is more than adequate to bring the doses to tolerance for $9 . \mathrm{GeV}$
operation. 


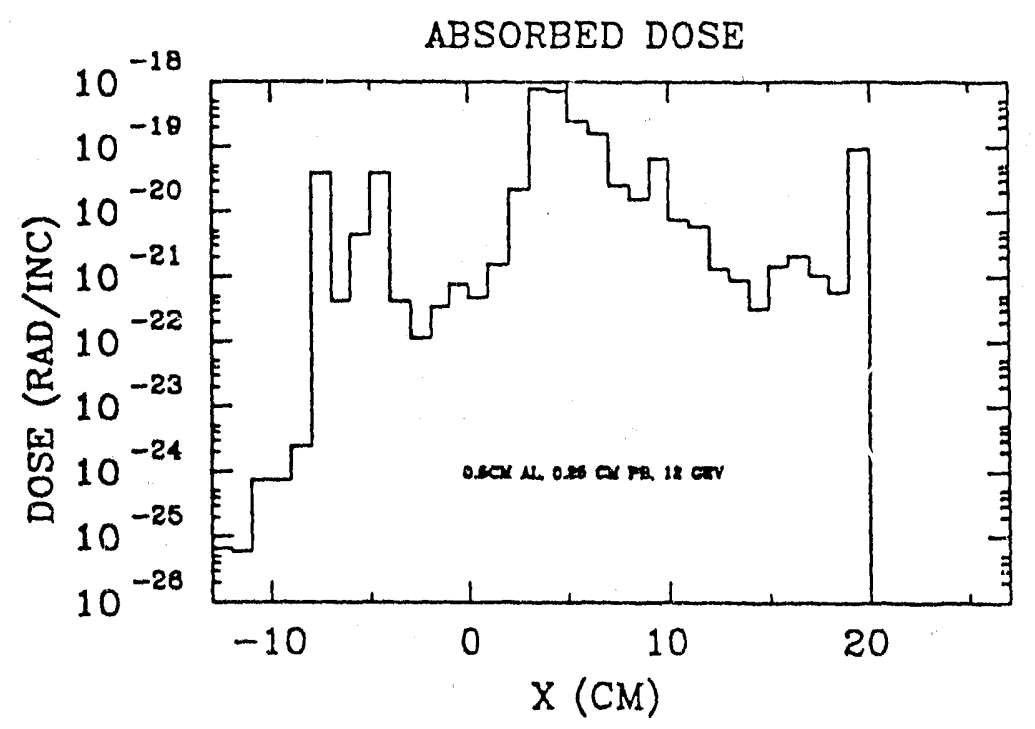

Figure 18. Dose through a $0.5-\mathrm{cm}$ thick $\mathrm{Al}$ beam pipe with a 0.25 -cm thick $\mathrm{Pb}$ liner for $12 \mathrm{GeV}$-operation.4

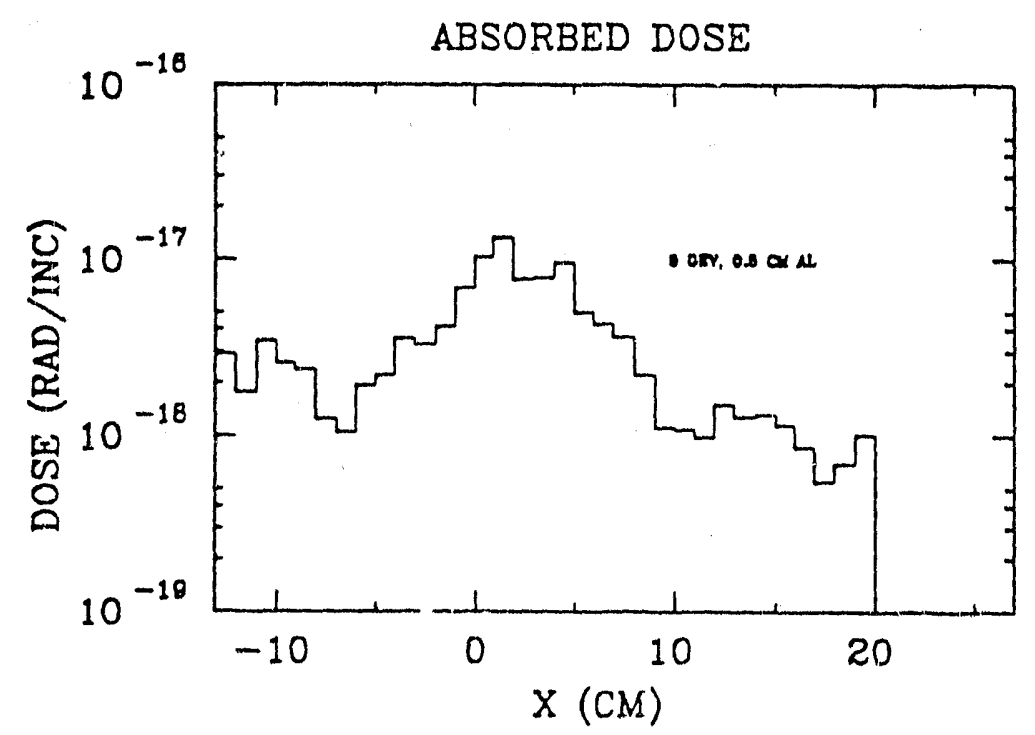

Figure 19. Dose through a $0.5-\mathrm{cm}$ thick Al beam pipe with no liner for $9 \mathrm{GeV}$-operation. 
For a copper pipe at $9-\mathrm{GeV}$ operation, a beam pipe $0.5-\mathrm{cm}$ thick is everywhere adequate as shown in Fig 20.

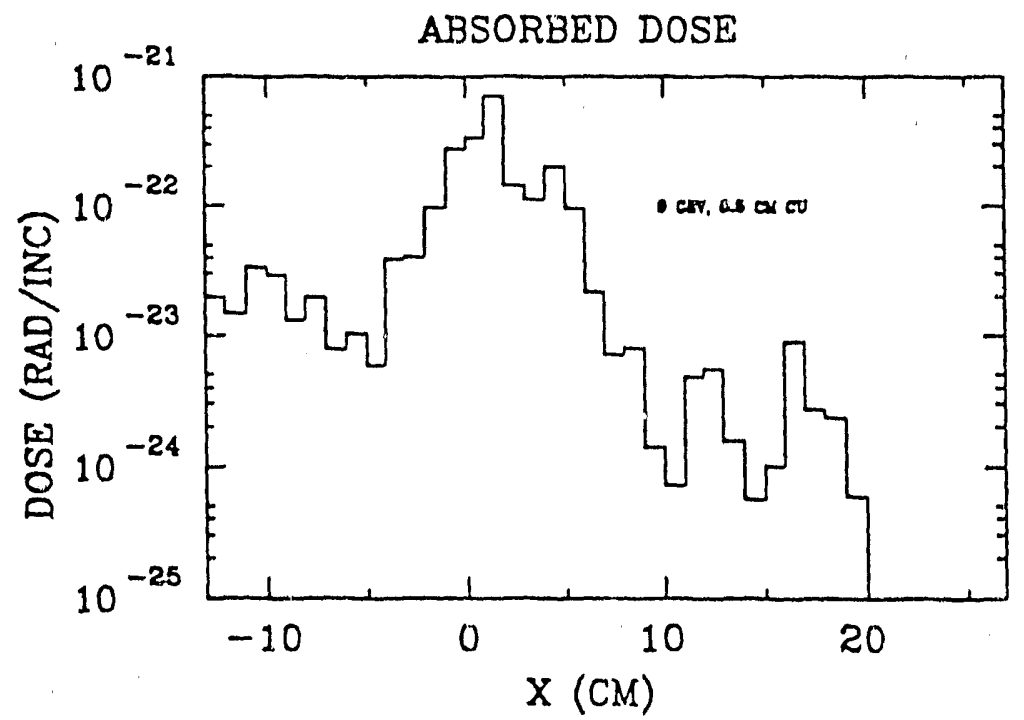

Figure 20. Dose through a $0.5-\mathrm{cm}$ thick $\mathrm{Cu}$ beam pipe with no liner for $9-\mathrm{GeV}$ operation.

\section{REFERENCES}

1. W.R. Nelson, G.J. Warren and R.L. Ford, "The Radiation Dose to the Coil Windings and the Production of Nitric Acid and Ozone from PEP Synchrotron Radiation", PEP Note PEP-109 (1975).

2. CERN 85-02, 1985.

3. B Factory Collider Note SLAC/LBL ABC-5, May 29, 1990

4. D. W. O. Rogers, "Fluence to Dose Equivalent Conversion Factors Calculated with EGS3 for Electrons from $100 \mathrm{keV}$ to $20 \mathrm{GeV}$ and Photons from $11 \mathrm{keV}$ to $20 \mathrm{GeV}$ ", H. Phys. 46 (1984) 891 

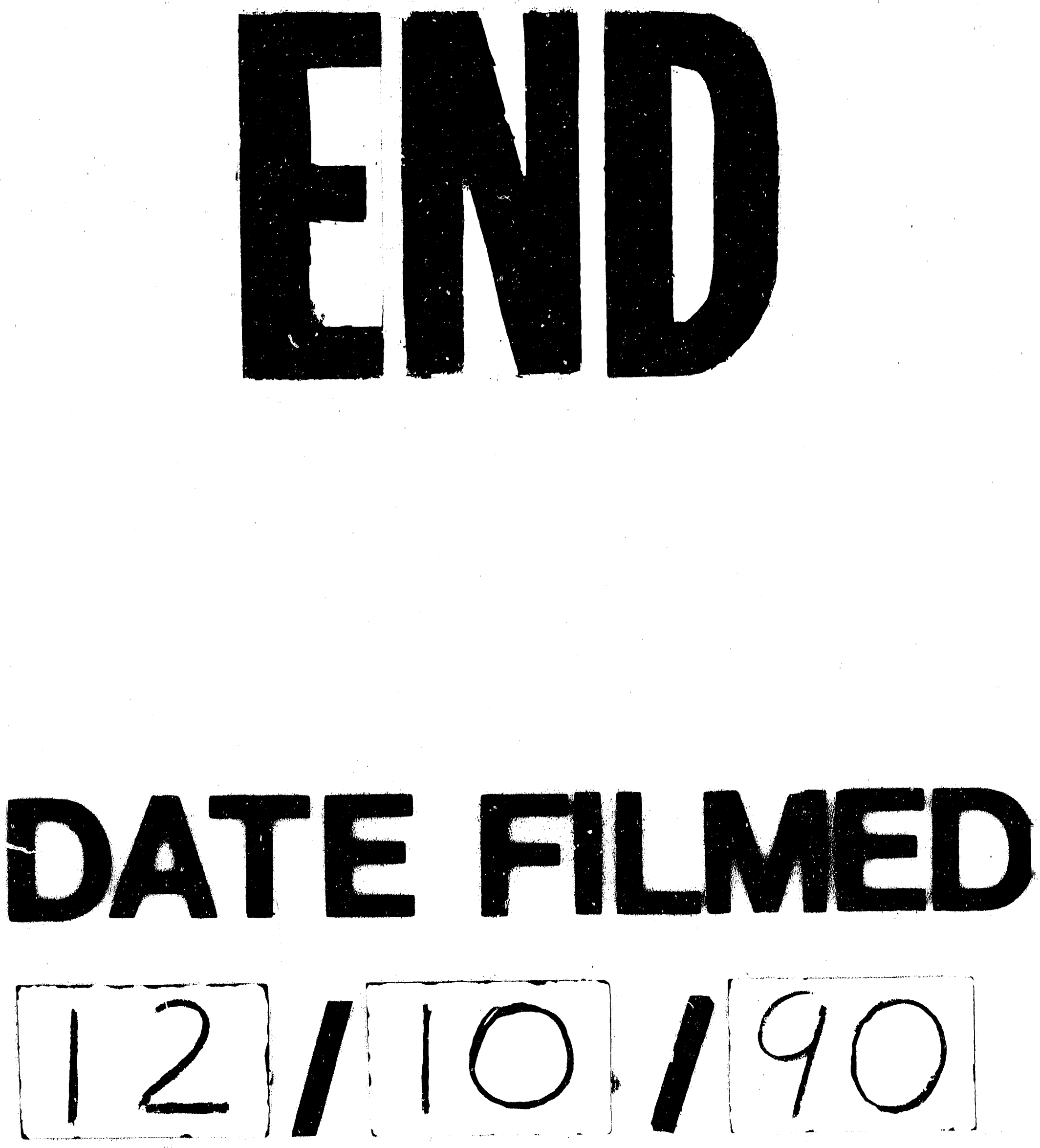
$\mathbf{L}$ 